\title{
Model-based inquiry vs. Traditional computer simulation-based instruction: Which can better help students construct the quantum-mechanical model of an atom?
}

\author{
Tugba Yuksel, ${ }^{1}$ N. Sanjay Rebello, ${ }^{2,1}$ and Lynn A. Bryan ${ }^{1,2}$ \\ ${ }^{I}$ Department of Curriculum and Instruction, Purdue University, 100 University St., West Lafayette, IN, 47907 \\ ${ }^{2}$ Department of Physics and Astronomy, Purdue University, 525 Northwestern Ave., West Lafayette, IN, 47907 \\ Research suggests that students' conceptual models play an essential role in their understanding. Therefore, \\ model-based inquiry has been considered as an instructional method in which learners have the opportunity \\ to actively build and use their models. In this study, we investigated students' model evolution during their \\ learning experience with model-based instruction. We analyzed students' model transition process as they \\ engaged with a sequence of activities supported with physical, computer-based, and mathematical models. \\ We compared the results with students' who received traditional computer-based instruction. Results show \\ that students who received model-based inquiry instruction increased the sophistication of their explanation \\ and gained more accurate understanding compared to traditional compute-based instruction group.
}

\section{INTRODUCTION}

Research has shown that teaching and learning of quantum mechanics faces fundamental challenges [1-5]. Due to the abstract nature of the topic, students who receive traditional instruction often struggle to construct quantum mechanical models of an atom [6-9]. Non-traditional strategies including inquiry-based learning, computer-based learning, and hands-on activities to facilitate learning of quantum mechanics have also been used with mixed results in facilitating students to learn the quantum mechanical model of an atom [10-13].

Modeling is considered an important scientific practice [14]. Models are used by scientists to describe complex phenomena, and in instruction to help students develop and communicate their understanding $[1,15]$. Learners construct or refine models by interacting with objects and phenomena, and integrating this information with prior knowledge $[16,17]$. So, it seems plausible that an approach that emphasizes modeling can better facilitate students to understand quantum mechanics concepts, particularly atomic structure since research has shown students' learning difficulties in this topic.

We examined learning of fundamental quantum mechanical concepts in two different conditions: using a model-based inquiry curriculum and traditional textbook instruction supported with computer-based simulations. Our research question is: How do these two strategies facilitate students' learning of the quantum mechanical model of an atom?

\section{METHODS}

\section{A. Context}

This study was conducted at a large U.S. Midwestern land grant university. We developed a learning module where different types of models (visual, physical, graphical and mathematical) were integrated in well-guided instruction. Students were prompted to think critically and construct their models of quantum phenomena. Model-based activities including computer simulations were designed to facilitate students' development of the models.

\section{B. Participants}

Seven freshmen and one sophomore physics or engineering major enrolled in second semester calculusbased physics participated in this research study. After initial screening, all participants completed a pre-questionnaire, which helped us evaluate students' initial models of atomic structure and other fundamental quantum concepts. Students' were assigned either to the treatment group which received the model-based learning module (MBLM) $(\mathrm{N}=4)$ or to the control group who received traditional computerbased simulation instruction (TCBS) $(\mathrm{N}=4)$. Although it was very difficult to match students' initial performance in such a small group, we tried to assign similar students to different groups.

\section{Study Design}

Before receiving the MBLM, participants received a set of questions including atomic structure, electron's motion and configurations. Students had the first 25-30 minutes to 
think about the answers and write them down. After the think-alone process, we started interviewing the participants so that they could elaborate their answers. During the intervention, MBLM students received a model-based learning module (MBLM), which included a series of lessons supported with modeling-based activities and embedded assessments to encourage them to articulate/improve their model-based reasoning and critical thinking. The modelbased activities consisted of physical models, computer simulations, and graphical and mathematical models. They were also given a booklet consisting of a collection of relevant chapters about the quantum mechanics topics in sequence.

The control group (TCBS) received the same set of computer-based simulations supported with the same text booklet, but they did not receive any model-based instruction. Both groups had worksheets for each activity in which to record their observations and interpretations. The total time for the study was four weeks. To keep the time spent on learning materials the same for both groups, the control group was asked to read related chapters and write a summary at the beginning of each lesson.

Both groups finished the activities related to atomic structure, energy levels and atomic spectra in four weeks (one two-hour long meeting each week). After completion of all the units, students took a post questionnaire followed by another interview. The pre- and post-interviews and all of the instruction were video and audio recorded as part of the data sources. One student in the MBLM group left the study after third week and did not take the post-questionnaire.

\section{Data Analysis}

In pre- and post-questionnaire, we asked students to give as much as detail as possible about the hydrogen atom. Specifically, we analyzed three aspects of the data (a) Atomic structure i.e. the physical arrangements of various parts of the atom (b) Electron behavior i.e. how electrons move and how they are arranged in a multi-electron atom (c) Energy levels and atomic spectra at their relationship.

The data were analyzed by using open coding [18]. Four model categories emerged: Discrete Entity (particle), hybrid, quantum-like and quantum. To ensure inter-rater reliability of the rubric and categorization of students' models, another expert researcher analyzed students' pre- and postquestionnaire models. A 100\% agreement was established after discussion.

\section{RESULTS}

Responses that we categorized as a discrete entity (particle) model demonstrated a conceptual understanding that carried many classical mechanics-based perspectives, such as planet-like orbits of electrons. Responses that we categorized as the hybrid model appeared to blend both quantum mechanical and classical mechanics concepts. Responses that we categorized as the quantum-like model had several aspects of the quantum nature of the atom, such as energy levels and transitions, except that they used the deterministic, rather than probabilistic perspective. Finally, responses that were categorized as the quantum model included the probabilistic perspective.

\section{A. Prevalence of Models}

Figure 1 shows the prevalence of various models in each group both pre and post-instruction. The data indicate that there was a greater prevalence of the quantum model in postinstruction for both groups, compared to pre-instruction. However, the hybrid and entity models were most prevalent in pre-instruction for both the groups.

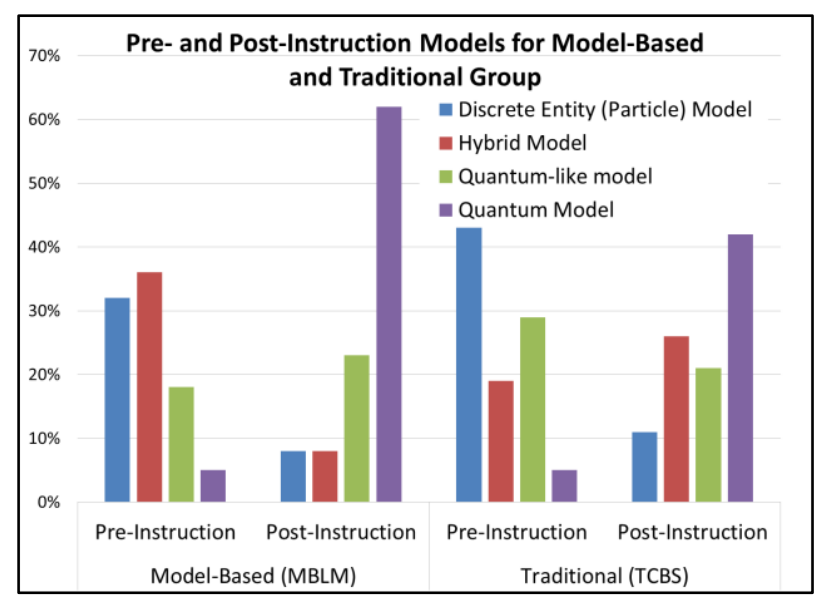

FIG 1.Pre- and post-Instruction models for both groups.

\section{B. Model Transitions}

An analysis of the prevalence of models does not show which students in each group actually changed their models. To ascertain which groups showed a transition in their models in each format of instruction, we analyzed each student separately. Figure 2 shows the transitions for the MBLM group and Fig. 3 shows the transitions for the TCBS group. 


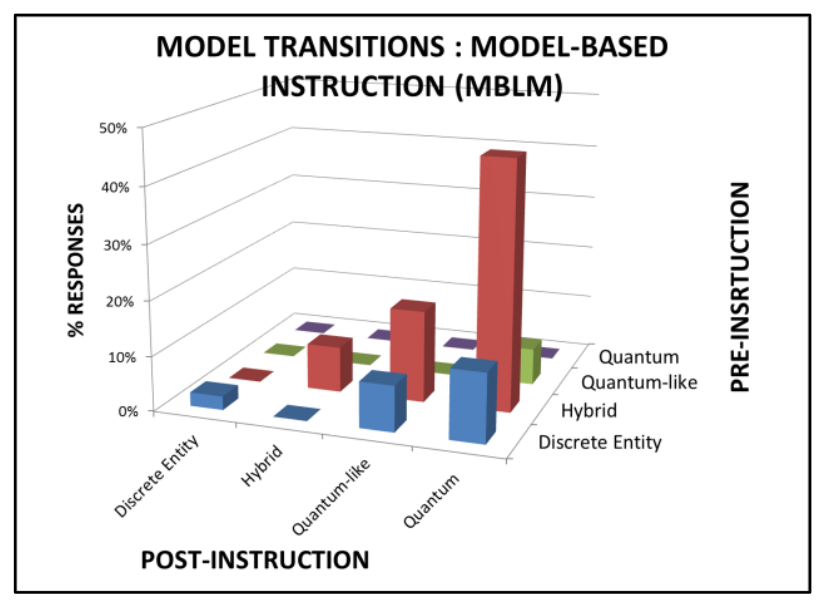

FIG 2. Model transitions in the MBLM group.

Figure 2 and Fig. 3, show the percentage of students' in the initial (pre-instruction) and final (post-instruction) stages are presented as categorized in the four types of model states. The data show that in the MBLM group, most transitions $(45 \%)$ into the quantum or quantum-like model state in postinstruction occurred from the hybrid model state in preinstruction. However, in the TCBS group, most transitions into the quantum model state $(22 \%)$ occurred from the quantum-like state in pre-instruction.

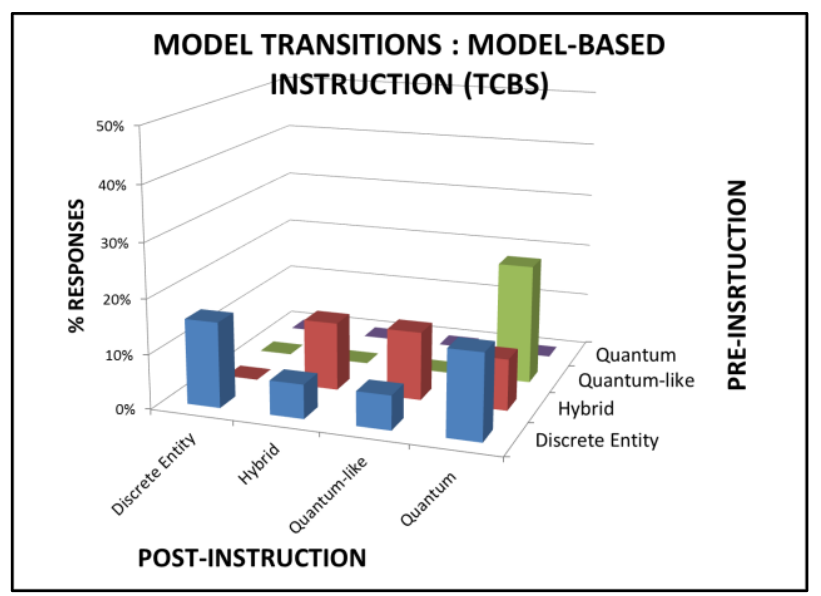

FIG 3. Model transition in the TCBS group.

These results seem to suggest that the model-based instruction was most beneficial to facilitate students whose responses were in the hybrid state, in that it facilitated their transition to the quantum model state. However, traditional instruction seems to favor students whose responses were already in the quantum-like model state, in that it facilitated their transition into the quantum model state. It must be noted, that these results are based on a rather small sample of eight students who participated in this study.

\section{Two Cases}

As exemplars from our study, we present two students' atomic structure model change as examples. Both students were selected because their performance was typical of students' performance in their assigned group. Mohan's (not real name) initial and final models are shown in Fig 4. He was in the MBLM group.

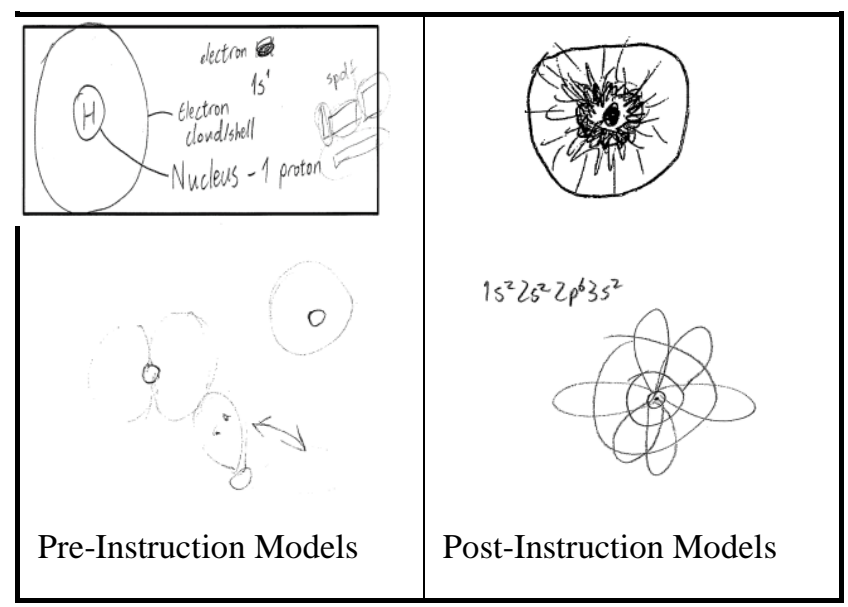

FIG 4. Mohan's pre- and post-instruction atomic models

Mohan's model (hybrid) prior to the intervention indicated patterns from both classical mechanics and quantum mechanics. While he mentioned about $1 \mathrm{~s}$ orbital for hydrogen atom, he did not state "orbitals" in his multielectron model. Although Mohan's attempt to depict $\mathrm{s}$ and $\mathrm{p}$ orbitals for multi-electron model illustrates his quantum mechanical perspective, locating electrons inside the orbitals showed his classic mechanical perspective. After instruction, Mohan shifted his model toward the quantum perspective and drew orbital representations for both hydrogen and multi-electron atoms. Moreover, he demonstrated probability density in hydrogen atom model.

Another student 'Thomas', was in the traditional instruction group. His responses initially demonstrate a hybrid model and remained as hybrid model after TCBS instruction. Figure 4 presents Thomas's initial and final hydrogen and multi-electron models. Thomas was one of the students who showed the wave nature of electron with a classical wave representation. His multi-electron atom model had three electrons and they were located in a sphere shaped "electron cloud". After instruction although he drew what 
appear to be the s and p orbitals for hydrogen atom, he also depicted the Bohr model of atom for multi-electron atom model.

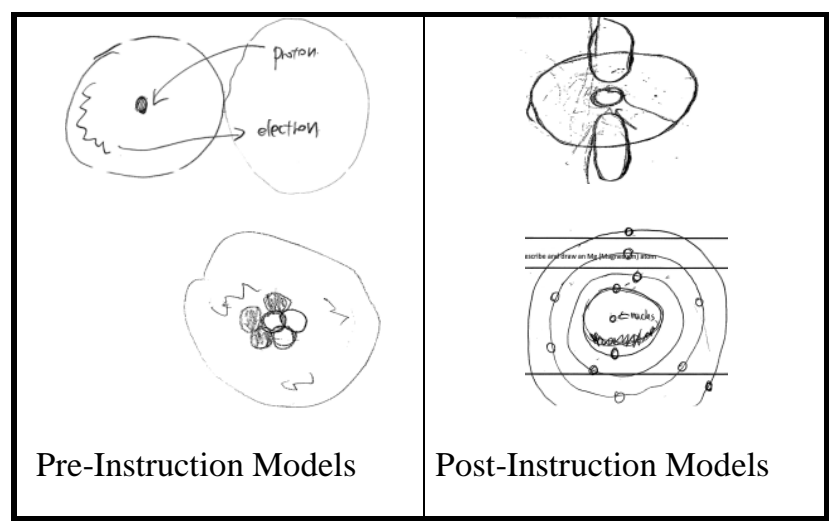

FIG 5. Thomas's pre- and post-instruction atomic models

\section{DISCUSSION}

In this paper, we have presented the results of our qualitative study of students' conceptual understanding and model-based reasoning about a fundamental quantum mechanics concept: the nature of an atom. Although many prior studies have focused on common misconceptions that students have in this area, few investigations have proposed an innovative approach to facilitate students in overcoming their learning challenges and addressing their difficulties with conceptualizing an atom. In our research, we aimed to provide a model-based learning environment that we believe helps students construct knowledge in a meaningful order, and evaluate, revise and refine existing models. [13, 15].

The findings of this study seem to indicate that students whose responses were in the hybrid model state were most amenable to transitioning to a quantum model state as a result of model-based instruction. This result can be explained in terms of the zone of proximal development (ZPD) [19]. It appears that model-based instruction seemed to expand the ZPD for students in the hybrid model state much more so than traditional computer-based instruction did in this study.

An important limitation of this study was clearly the very small sample size of four participants in each group. The small number of participants made it difficult for the researchers to create matched groups. Thus the individual participants in the two conditions started out with very different models of the atom before they participated in the intervention due to the study.

\section{ACKNOWLEDGEMENTS}

We wish to thank the participants for volunteering their time to participate in the study.
[1] J. Clement, Am. J. Phys. 50, 66-71 (1982).

[2] R. Olsen, Int. J. Sci. Ed., 24, 565-574 (2002).

[3] M. Wittmann, R. Steinberg and E. Redish, Am. J.Phys. 70, 3, (2002).

[4] G. Tsaparlis and G. Papaphotis, Int. J. Sci. Ed., 31, (2009).

[5] C. Singh, Am. J. Phys,.69, 8, (2001); 76, 4, (2008).

[6] M. Euler et al. Presented at the annual meeting of NARST, 1999

[7] I. Johnston, K. Crawford and P. Fletcher, Int. J. Ed. 20, 4, (1998).

[8] H. Fischler and M. Lichtfeldt, Int. J. Sci. Ed., 14, p.181, (1992)

[9] D. Zollman, Presented at the annual meeting of NARST, 1999

[10]D. Zollman et al., Am. J. Phys. 70, 252 (2002).
[11] R. Justi, and J. Gilbert, Int. J. Sci. Educ., 22, 993 (2000).

[12] R. E. Stake, The art of case study research, (Sage, 1995).

[13] B.C. Buckley, Int. J. Sci. Educ., 22, 895 (2000).

[14] National Research Council. Washington, DC: The National Academies Press (2007); (2002).

[15] J. D. Gobert and B. C. Buckley, Int. J. Sci. Educ., 22, 891 (2000).

[16] P. Johnson-Laird, Mental Models, 6th ed. (Harvard Univ. Press, Cambridge [U.A.], 1995).

[17]E. Von Glasersfeld, Radical constructivism: A way of knowing and learning. (London: Falmer,1995).

[18] A. Strauss and J. Corbin, J., Basics of qualitative research (Vol. 15). (Newbury Park, CA: Sage, 1990)

[19] L.S. Vygotsky, Mind in society, Cambridge, MA: Harvard University Press (1978). 